\title{
Towards Future Circular Colliders *
}

\author{
R. Tomás $\dagger$, M. Benedikt, M. Hofer, J. Keintzel and F. Zimmermann \\ CERN, Geneva, Switzerland \\ E-mail: rogelio.tomas@cern.ch, michael.benediktecern.ch, \\ michael.hofer@cern.ch, jacqueline.keintzel@cern.ch and \\ frank.zimmermann@cern.ch
}

The international Future Circular Collider (FCC) study, launched in 2014, is finalizing a multivolume conceptual design report. The FCC develops high-energy circular collider options based on a new $100 \mathrm{~km}$ tunnel. Long-term goal is a $100 \mathrm{TeV}$ center-of-mass proton-proton collider (FCC-hh). The study includes a high-luminosity electron-positron collider (FCC-ee) as a possible first step, and it also examines lepton-hadron scenarios (FCC-he). In addition, the FCC study includes the design of the High Energy LHC (HE-LHC), housed in the LHC tunnel, and based on the same high-field magnet technology as the FCC-hh. The FCC study further includes an elaboration of the physics cases, including for heavy-ion collisions, and detector concepts, as well as staging and implementation scenarios. This report summarizes the study achievements with special emphasis on the HE-LHC.

Sixth Annual Conference on Large Hadron Collider Physics (LHCP2018)

4-9 June 2018

Bologna, Italy

${ }^{*}$ This work was supported by the European Commission under the HORIZON 2020 projects EuroCirCol, grant agreement no. 654305; EASITrain no. 764879; and ARIES no. 730871.

${ }^{\dagger}$ Speaker. 


\section{Motivation, History, and Scope}

The LHC design was launched in 1983, 35 years ago. The physics programme of the LHC [1] and its high-luminosity upgrade, the HL-LHC [2,3], will extend through the 2030's. In view of these time scales, the 2013 Update of the European Strategy for Particle Physics requested preparations for a post-LHC collider at CERN [4]. European studies of highest-energy highestluminosity large circular colliders had started already a few years earlier, in 2010-2012, for both leptons [5, 6] and hadrons [7, 8, 9], under the names LEP3/TLEP and VHE-LHC, respectively. In early 2014 these efforts were combined and expanded as global Future Circular Collider (FCC) study $[10,11]$.

The center-of-mass (c.o.m.) energy reach of a hadron collider is directly proportional to the dipole magnetic field $B$ and to the bending radius $\rho$, or machine circumference. Dipole magnets with a field of $16 \mathrm{~T}$ together with a ring circumference of about $100 \mathrm{~km}$ result in a c.o.m. energy of $100 \mathrm{TeV}$, an order of magnitude above the LHC. This goal for a future circular hadron collider (FCC-hh) defines the overall infrastructure requirements for the FCC accelerator complex. The FCC study scope also includes the design of a high-luminosity $\mathrm{e}^{+} \mathrm{e}^{-}$collider (FCC-ee) operating at c.o.m. energies of $90-365 \mathrm{GeV}$, as a possible first step, as well as a proton-electron collision option (FCC-he) at one interaction point, where a $60 \mathrm{GeV}$ electron beam from an energy recovery linac would be collided with one of the two $50 \mathrm{TeV}$ proton beams circulating in the FCC-hh. The design of a higher-energy hadron collider in the LHC tunnel with a c.o.m. energy around $27 \mathrm{TeV}$, based on FCC-hh magnet technology — the so-called High-Energy LHC (HE-LHC) — is yet another part of the FCC study. The FCC study comprises accelerator design, technology development, detector design, physics cases, conventional infrastructure, implementation scenarios and cost estimates, for all collider scenarios.

\section{Physics and Design Targets}

The lepton collider FCC-ee will explore the $10-100 \mathrm{TeV}$ energy energy scale via couplings with precision measurements [12]. It will yield a 20-50 fold improved precision for many electroweak quantities (equivalent to a factor 5-7 in energy), such as $m_{Z}, m_{W}, m_{t}, \sin ^{2} \theta_{\mathrm{W}}^{\text {eff }}, R_{b}, \alpha_{\mathrm{QED}}\left(m_{Z}\right)$, $\alpha_{s}\left(m_{Z}, m_{W}, m_{t}\right)$, Higgs boson and top quark couplings. To accomplish these goals, the machine is designed for highest possible luminosities at four working points $(Z, W W, Z H$ and $t \bar{t})$.

The FCC-hh will provide collisions at highest c.o.m. energy for direct production up to 20 $30 \mathrm{TeV}$. There will also be huge production rates for single and multiple production of SM bosons $(H, W, Z)$ and quarks [13]. The machine is designed for $100 \mathrm{TeV}$ c.o.m. energy with an integrated luminosity of $20 \mathrm{ab}^{-1}$ over 25 years.

The HE-LHC design aims at approximately doubling the LHC collision energy with FCC-hh $16 \mathrm{~T}$ magnet technology in the $26.7 \mathrm{~km}$ LHC tunnel. The c.o.m. energy of $27 \mathrm{TeV}$ is obtained by scaling with the magnetic field from the LHC's $14 \mathrm{TeV}$ with $8.33 \mathrm{~T}$ dipole field. The HELHC target luminosity is more than $10 \mathrm{ab}^{-1}$ over 20 years. The HE-LHC machine is designed within the constraints of the existing LHC infrastructure, and it incorporates both HL-LHC and FCC technologies. 
FCC-ee and FCC-hh designs are well advanced as presented in [14]. In the following we focus in the recent HE-LHC design progress.

\section{High-Energy LHC}

The HE-LHC must be installed in the existing LHC tunnel, with an inner diameter of only $3.8 \mathrm{~m}$, compared with $5.5 \mathrm{~m}$ for the FCC. These space limitations result in significant constraints on the HE-LHC machine layout, magnets which must be compact and curved, and on the HE-LHC cryogenics system, which needs to be more powerful than the LHC one. A large variety of layouts has been explored for the HE-LHC [15] concluding with the two most promising options: $23 \times 90$ (23 cells per arc with $90^{\circ}$ phase advance) and 18x90. A sketch of the two cells is shown on Fig. 1.
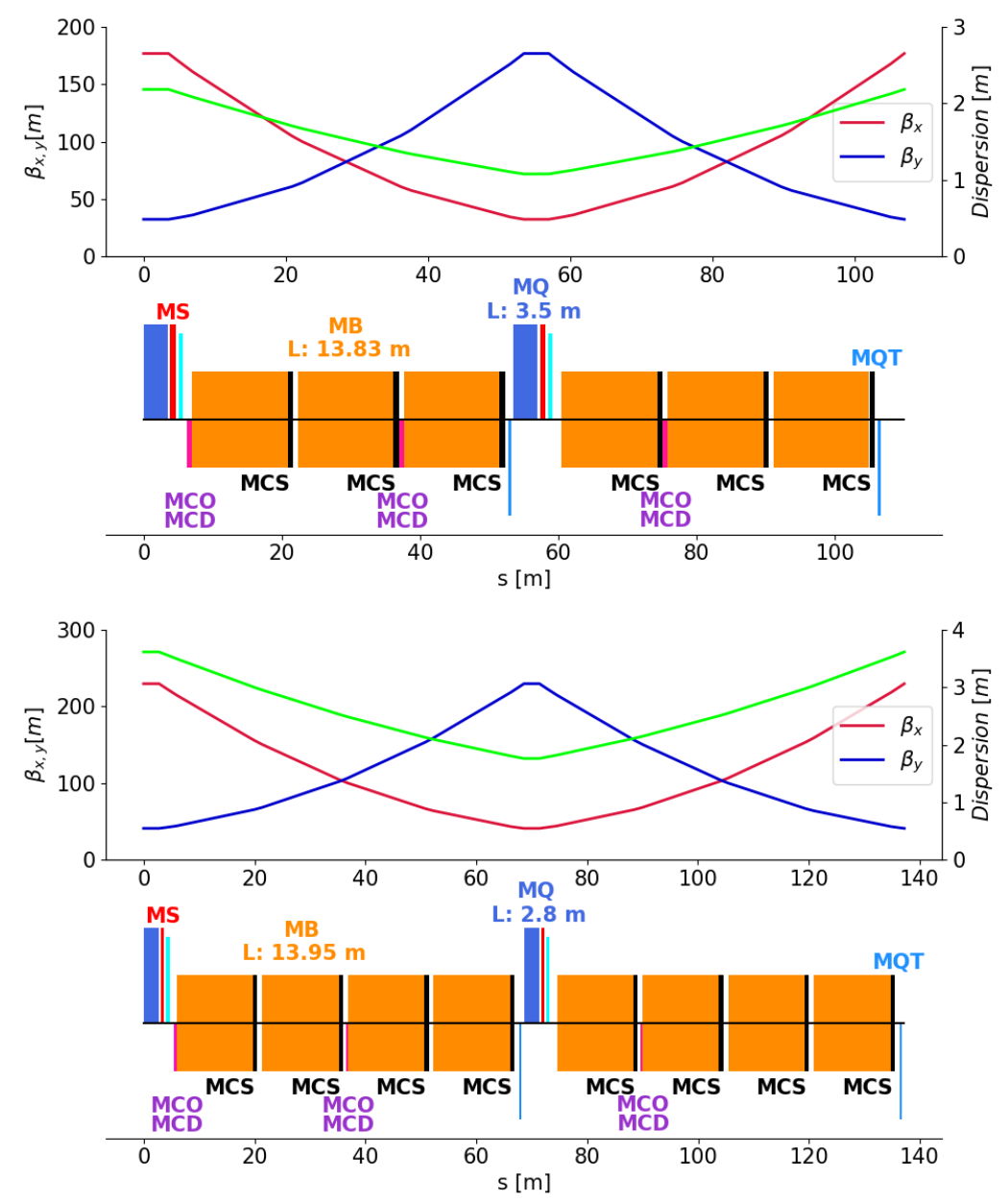

Figure 1: Optics functions and layout configuration of the two HE-LHC design options. Top: 23x90 cell. Bottom: 18x90 cell. Dipoles are shown in orange, quadrupoles in blue and sextupoles in red.

The horizontal deviation of the machine position with respect to the LHC is shown in Fig. 2 for both options. The configuration $23 \times 90$ only deviates by few $\mathrm{cm}$ as it has the same number of arc cells as the LHC. The option $18 \times 90$ has a peak deviation of about $9 \mathrm{~cm}$ and it is currently being investigated if this deviation is acceptable or it can be further reduced. 


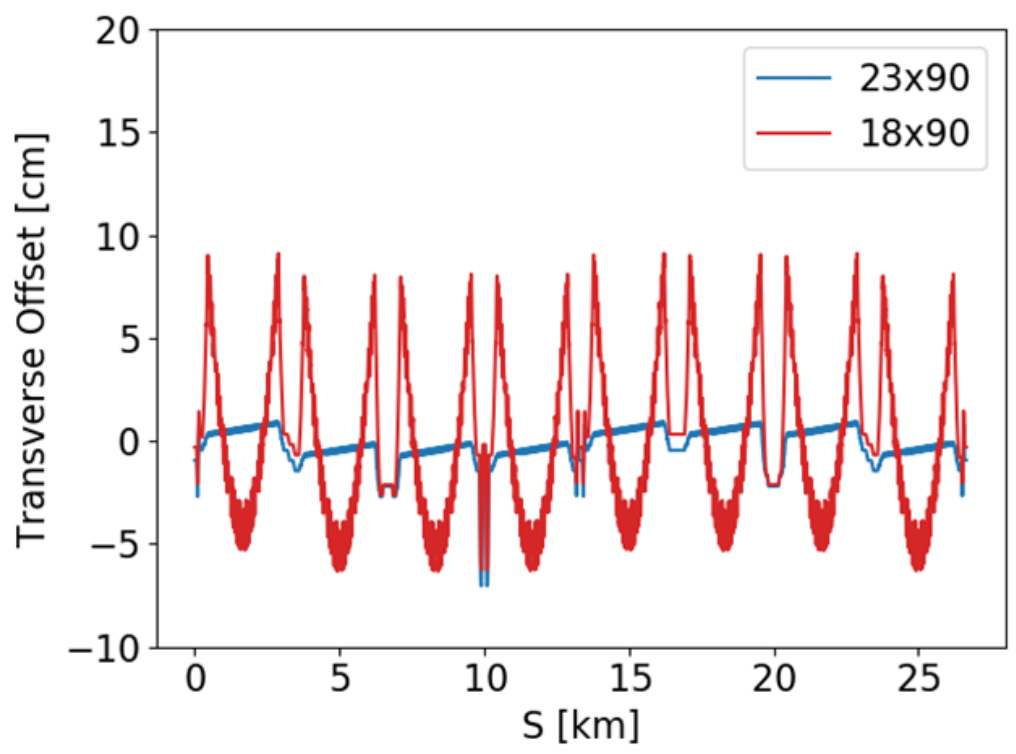

Figure 2: HE-LHC horizontal deviation with respect to the LHC for the two lattice options: $23 \times 90$ and $18 \times 90$.

The $18 \times 90$ option has 8 dipoles per cell and a higher filling factor than the $23 \times 90$ one, implying a higher energy reach. Actually assuming $16 \mathrm{~T}$ dipoles the $18 \mathrm{x} 90$ option can reach $27 \mathrm{TeV}$ c.o.m. energy while the $23 \times 90$ one stays at $26 \mathrm{TeV}$. At injection energy the situation is reversed and the $23 \times 90$ optics features a smaller transverse beam size and therefore larger physical and dynamic apertures for the same beam pipe and magnet designs. The 23x 90 design could have a beam stay clear of $10 \sigma$ at an injection energy of about $600 \mathrm{GeV}$, while the $18 \times 90$ design would need $800 \mathrm{GeV}$. The advantage of $600 \mathrm{GeV}$ is that the current LHC injector, the SPS, would only require upgrading half of the dipoles with superconducting ones. Current quadrupoles and sextupoles are sufficiently strong to reach $600 \mathrm{GeV}$ with an integer tune of 20. This partial upgrade of the SPS was already considered in 1972 [16]. Further studies are being pursued to find solutions that would allow to inject from the current SPS at $450 \mathrm{GeV}$.

Particle stability at injection is a great concern due to the required large energy swing of the dipole magnets and poor field quality at injection. Various approaches are being explored to maximize field quality and mitigate its impact on beam stability. Small filament size of $20 \mu \mathrm{m}$ greatly lowers the field errors at injection related to persistent currents. A further improvement of the field quality at injection is expected from the addition of artificial pinning centers (APCs). The APCs decrease the critical current at low field levels and thereby the strength of the persistent current effects. After magnets are manufactured the field quality can be measured and dipoles could be placed in the accelerator according to their sextupolar component to maximize the particle phase-space stability region, called Dynamic Aperture (DA). Assuming $20 \mu \mathrm{m}$ filament size and perfect APC the DA for the two lattice options with and without sorting is shown on Fig. 3 for three possible energies: $450 \mathrm{GeV}, 900 \mathrm{GeV}$ and $1.3 \mathrm{TeV}$. Former DA studies can be found in [18, 19].

Targeting a DA above $8 \sigma$ would allow the $23 \times 90$ design to run at any energy equal or above $450 \mathrm{GeV}$ while the $18 \times 90$ option would need injection energies equal or above $900 \mathrm{GeV}$. 


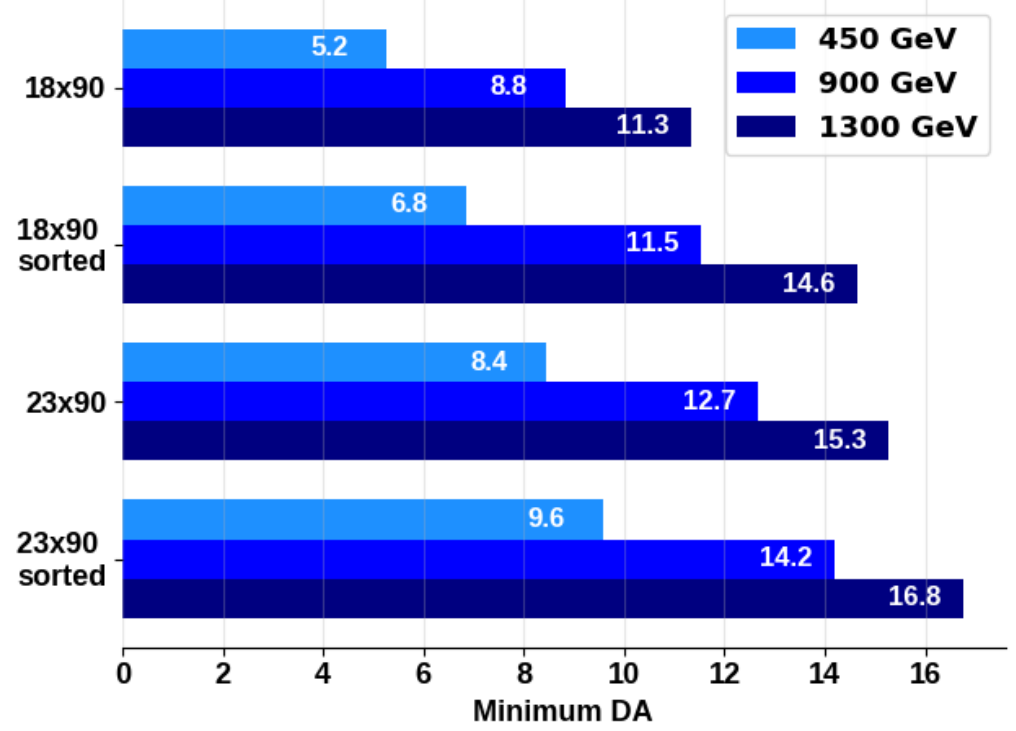

Figure 3: HE-LHC DA for magnets with $20 \mu \mathrm{m}$ filament and perfect artificial pinning centers with and without sorting for three different energies.

\subsection{HE-LHC performance}

The current baseline HE-LHC foresees a $\beta^{*}$ at the IP of $45 \mathrm{~cm}$, a bunch population of $2.2 \times 10^{11}$ protons, a normalized emittance of $2.5 \mu \mathrm{m}$ and operation with crab cavities to recover head-on collisions [20]. Initial luminosity of about $15 \times 10^{34} \mathrm{~cm}^{-2} \mathrm{~s}^{-1}$ and 430 events per bunch crossing are expected. After 5 hours in collisions the luminosity decreases by about a factor 3 since both bunch population and average emittance decrease by a factor 3 . An integrated yearly luminosity of about $470 \mathrm{fb}^{-1}$ can be achieved with 160 days of operation and $75 \%$ availability.

The $\beta^{*}$ of $45 \mathrm{~cm}$ is decided by triplet aperture and required crossing angle in the beginning of the fill. Since emittance reduces significantly it is conceivable to squeeze $\beta^{*}$ while keeping beam divergence at the IP constant. Keeping also constant crossing angle ensures that no extra aperture would be required in the triplets. This mode of operation could increase integrated yearly luminosity up to $590 \mathrm{fb}^{-1}$. The final $\beta^{*}$ would be around $15 \mathrm{~cm}$. A comparison of the instantaneous luminosity and other beam parameters during the fill for both baseline and constant divergence scenarios is shown in Fig. 4. The same simulation code as in [21] is used.

\section{Construction and Schedule}

Following a geological review an optimized baseline tunnel was established, with the lowest risk, the fastest and cheapest construction, and suitable locations for large-span caverns (the most challenging structures).

All surface and underground structures can be constructed within 6.5-7 years. Already 5 years after groundbreaking, the first FCC tunnel sectors would be ready for the installation of technical infrastructure. 

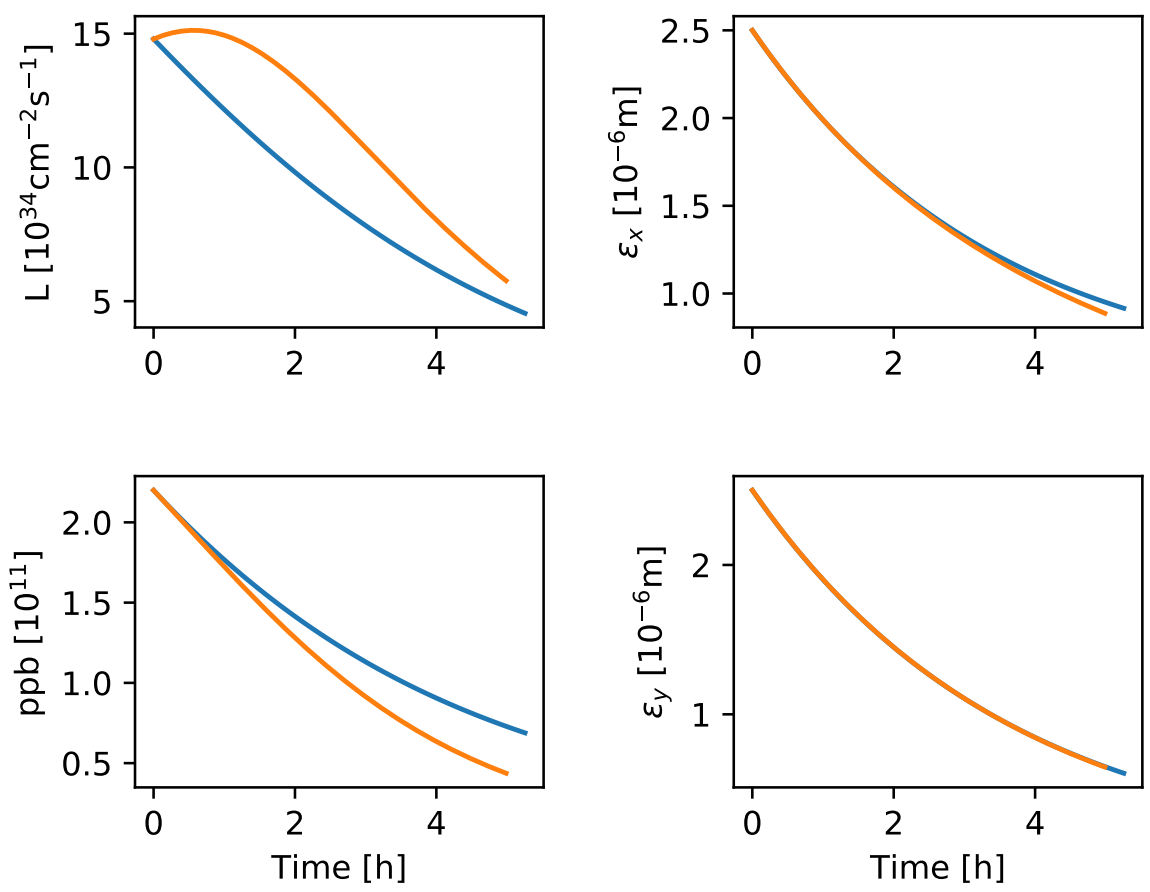

Figure 4: Simulated instantaneous luminosity, bunch intensity and horizontal and vertical emittances during a physics fill for both baseline (blue) and constant divergence (orange) HE-LHC scenarios at $27 \mathrm{TeV}$.

The first physics could be expected around 2040-45, for any of the three collider scenarios considered, in the case of FCC-ee about 5 years before FCC-hh.

\section{Summary and Outlook}

The FCC-ee and FCC-hh accelerator designs are ready for the conceptual design report (CDR). Recent developments of the HE-LHC design have been fundamental to identify the most promising lattice options for the CDR. A worldwide R\&D program is in place, on efficient high-field magnets, $\mathrm{Nb}_{3} \mathrm{Sn}$ superconductor, and on highly efficient SC RF. The international FCC collaboration is growing steadily. By now 124 institutes and 30 companies from 32 countries are participating. The collaboration is presently focusing on the completion of the conceptual design report (CDR). Prototyping and validation of key technical components are underway. The next phase of the FCC study, from 2019 to 2023, will focus on the implementation plan, and on the further development of key technologies, especially the high-field magnets.

\section{Acknowledgement}

This report summarizes results from the international FCC collaboration. Our warm thanks go to all collaboration members for their excellent, enthusiastic work and for many inspiring discussions. We are grateful to the colleagues who contributed the graphics of this article. 


\section{References}

[1] O. Brüning et al. (eds.), "LHC Design Report, Volume v.1: The LHC Main Ring", CERN-2004-003-V-1 (2004).

[2] G. Apollinari et al., "High-Luminosity Large Hadron Collider (HL-LHC): Technical Design Report V. 0.1”, CERN Yellow Report CERN-2017-007-M (2017).

[3] L.E. Medina Medrano et al., "New HL-LHC Baseline and Performance at Ultimate Energy", IPAC'18, Vancouver.

[4] European Strategy Session of Council, 30 May 2013, CERN-Council-S/106 (2013).

[5] A. Blondel, F. Zimmermann, "A High Luminosity $\mathrm{e}^{+} \mathrm{e}^{-}$Collider in the LHC Tunnel to Study the Higgs Boson," 12 December 2012, arXiv:1112.2518 [hep-ex] .

[6] EuCARD LEP3 workshop, 18 June 2012, http : / / indico. cern. ch/ event/193791; 2nd EuCARD LEP3 workshop, 23 October 2012, http : / / indico. cern. ch/ event/211018; 3rd EuCARD TLEP3 workshop, 10 January 2013, http: / / indico.cern.ch/event/222458; 4th EuCARD TLEP workshop, 4-5 April 2013, http: / / indico. cern. ch/event/240814.

[7] E. Todesco and F. Zimmermann (eds.), Proc. EuCARD-AccNet-EuroLumi Workshop: The High-Energy Large Hadron Collider - HE-LHC10, Malta, 14-16 October 2010, CERN-2011-003, arXiv:1111.7188 [physics.acc-ph].

[8] J.A. Osborne, C.S. Waaijer, "Pre-Feasability Assessment for an $80 \mathrm{~km}$ Tunnel Project at CERN", Contribution to the Update of the European Strategy for Particle Physics, No. 165, 27 July 2012.

[9] Joint Snowmass-EuCARD/AccNet-HiLumi meeting 'Frontier Capabilities for Hadron Colliders 2013' a.k.a. EuCARD VHE-LHC Day, CERN, 21-22 February 2013, http://indico.cern.ch/event/223094.

[10] M. Benedikt and F. Zimmermann, "Towards Future Circular Colliders", Journal of the Korean Physical Society 69 (2016) 893.

[11] http://cern.ch/fcc.

[12] H. Bicer et al., "First Look at the Physics Case of TLEP”, JHEP 01 (2014) 164.

[13] M. Mangano (ed.), "Physics at the FCC-hh, a $100 \mathrm{TeV}$ pp collider", CERN-2017-003-M and arXiv:1710.06353 (2017).

[14] M. Benedikt et al., "FCC: Colliders at the energy frontier", IPAC'18, Vancouver.

[15] J. Keintzel, “Arc Cell Options for the HE-LHC”, CERN-ACC-2018-0020.

[16] "The $300 \mathrm{GeV}$ Programme", CERN/1050, 1972. http: / / cds . cern. ch/record/2 4016

[17] D. Amorim et al., "High-Energy LHC Design,” presented at IPAC'18, Vancouver.

[18] M. Hofer et al., "Integrated Full HE-LHC Optics and Its Performance”, IPAC'18, Vancouver.

[19] Y. Nosochkov et al, “Optimized arc optics for the HE-LHC”, IPAC'18, Vancouver.

[20] L. van Riesen-Haupt et al., "Experimental interaction region optics for the High Energy LHC", IPAC'18, Vancouver.

[21] L. E. Medina Medrano et al., "Assessment of the performance of High Luminosity LHC operational scenarios: integrated luminosity and effective pile-up density", Canadian Journal of Physics, Vol. 0 (2018). 